\title{
SELETIVIDADE NA FLOTAÇÃO CATIÔNICA REVERSA DE MINÉRIO DE FERRO
}

\author{
M.A.M. AGUIAR ${ }^{*}$, R. M. FURTADO, A. E. C. PERES \\ Centro Federal de Educação Tecnológica de Minas Gerais \\ dodora_aguiar@hotmail.com*
}

Submetido 20/11/2017 - Aceito 24/11/2017

DOI: $10.15628 /$ holos.2017.6582

\section{RESUMO}

A amina, quando adicionada em solução aquosa contendo quartzo, adsorve-se na interface sólido-líquido em determinado $\mathrm{pH}$, tornando as partículas de quartzo hidrofóbicas. Tal hidrofobicidade pode ser afetada pela adição de amido, que é capaz de adsorver-se mutuamente com a amina, possivelmente devido à formação de um complexo helicoidal entre os reagentes, denominado de clatrato. Assim, o quartzo readquire caráter hidrofílico, por a amina ser mascarada pelo invólucro de amido. Foram realizados ensaios de microflotação e eletroforese. Nos ensaios de microflotação, foi estudada a flotabilidade da hematita e quartzo com os reagentes eteramina e os amidos maisena e amidex 4001. A maisena promoveu diminuição da flotabilidade do quartzo de $95 \%$ para $35 \%$ com aumento da dosagem de amina. O amidex não mostrou essa tendência nas condições estudadas. A depressão do quartzo foi favorecida pelo aumento nas dosagens tanto de maisena quanto de amina, e por condições básicas de $\mathrm{pH}$. Os ensaios de eletroforese, para determinação do potencial zeta, foram realizados com amina e amina e maisena em concentrações de 80 $\mathrm{mg} / \mathrm{L}$, nas quais houve evidência da formação de clatratos. O potencial zeta apresentou valores menos negativos em $\mathrm{pH}$ superior a 7, quando comparado com o potencial zeta de quartzo condicionado apenas com amina. Por fim realizou-se ensaios com amina e amidex em concentrações de $80 \mathrm{mg} / \mathrm{L}$, nas quais não foi observada variação significativa do potencial zeta quando comparado com potencial zeta de quartzo com amina nas mesmas condições.

PALAVRAS-CHAVE: flotação, minério de ferro, clatratos.

\section{SELECTIVITY IN THE REVERSE CATIONIC FLOTATION OF IRON ORE}

\begin{abstract}
Amines adsorbs at the solid-liquid interface when added to aqueous solution which contains quartz particles, under certain $\mathrm{pH}$ conditions, rendering these particles hydrophobic. However, this hydrophobicity may be affected by the addition of starch into the system, which is capable of adsorbing mutually with amine due to the formation of a helical complex between the reagents, called clathrate. Therefore, the quartz particles reacquire a hydrophilic surface since the amine is hidden by the starch envelopment. Microflotation and electrophoresis experiments were conducted with hematite and quartz and the reagents etheramine (frother and quartz collector) and corn starch and amidex 4001 (hematite depressants). The corn starch
\end{abstract}

promoted a decrease in the floatability of quartz, dropping from $95 \%$ to $35 \%$ as amine dosage was increased. amidex did not show this trend at any condition investigated. Quartz depression was favored by the increase in the dosage of both amine and corn starch and by alcaline $\mathrm{pH}$ conditions. The electrophoreses experiments for zeta potential measurements were conducted with amine and amine and corn starch at $80 \mathrm{mg} / \mathrm{L}$, there was evidences of the formation of clathrates were observed. In these conditions, higher zeta potential values were obtained when compared with the results obtained for quartz with amine only.

KEYWORDS: flotation, iron ore, clathates. 


\section{INTRODUÇÃO}

O sistema de concentração de minério de ferro consagrado no Brasil desde 1977 é a flotação catiônica reversa, na qual deprime-se os minerais-minério de ferro e flota-se a ganga silicática. Em termos de reagentes, o amido de milho é utilizado como depressor de óxidos e hidróxidos de ferro e a amina como coletor de quartzo e espumante. O condicionamento da polpa de minério de ferro, caso realizado diretamente com amina, tornaria tanto as partículas de minerais de ferro quanto as de quartzo hidrofóbicas. Isto impossibilitaria a concentração por flotação. Por essa razão, a polpa deve ser condicionada previamente em solução de amido gelatinizado, que se adsorve seletivamente sobre as partículas de hematita, tornando-as imunes à adsorção da amina. Posteriormente, condiciona-se a polpa em solução de amina. Desse modo, ao final, espera-se que a hematita permaneça hidrofílica e o quartzo torne-se hidrofóbico. Devido à alta demanda por ferro, os depósitos ferríferos brasileiros de altos teores têm se tornado escassos, sendo necessário iniciar a explotação de depósitos minerais com teores de ferro menores. O consumo de amina tende a aumentar com o aumento da quantidade de ganga silicática processada e diminuição da granulometria das partículas. Há indícios de que, em certas condições (composição do amido, granulometria, $\mathrm{pH}$ e dosagem de reagentes), o amido e o coletor (amina, neste caso) interagem, formando os clatratos - termo utilizado para denominar o composto molecular formado pelo alojamento das moléculas de amina no interior das moléculas de amido, que resulta na depressão de quartzo. A formação de clatrato pode justificar o indesejado aumento do teor de $\mathrm{SiO}_{2}$ no concentrado, o que compromete a qualidade do mesmo.

Interações entre amido e coletor vêm sendo relatadas por diversos pesquisadores (Takagi \& Isemura, 1960; Somasundaran, 1969; Partridge \& Smith, 1972; Hendrix \& Smith (1972); Somasundaran \& Cleverdon, 1985; Svensson Gundmundsson, \& Eliasson, 1996). Takagi \& Isemura (1960) mostraram evidências dessa interação analisando os efeitos do dodecilsulfonato de sódio em soluções de amilose e amilopectina, tendo relacionado essa interação à formação de um complexo entre o agente ativo de superfície e a amilose através de sua estrutura helicoidal. Somasundaran (1969) mostrou que o amido diminui o caráter hidrofóbico da calcita através de interações com o oleato, as quais denominou de clatrato. Partridge \& Smith (1972) e Hendrix \& Smith (1972) atribuíram a formação do complexo aos seguintes mecanismos: atração eletrostática entre + 3RNH e a molécula de amido carregada negativamente, forças de Van der Waals e ligações de hidrogênio. Somasundaran \& Cleverdon (1985) estudaram interações entre polímero e surfatante catiônico e aniônico em quartzo através de técnicas de adsorção, potencial zeta e flotação. Em pH 6,5 o polímero deprimiu o quartzo, sem diminuir adsorção da amina. 0 potencial zeta apresentou valores característicos do amido. Por volta do $\mathrm{pH} 10$, em que a amina tem maior atividade superficial, a adsorção do polímero foi negligenciável. Svensson et al. (1996) estudaram a ligação do dodecilsulfato (SDS) com amilose e amilopectina extraídas do amido de batata. Estimaram que a quantidade de ligações SDS/amilose é muito maior que a quantidade SDS/amilopectina e, no caso da amilose, a atividade superficial do complexo formado aumentou com altas concentrações de surfatante. O complexo SDS/amilose ocorreu através do alojamento das moléculas de SDS no interior das hélices da amilose. 
O efeito das interações amido/amina em escala de bancada foi estudado por Lima, Valadão e Peres (2013). Os autores realizaram ensaios de flotação catiônica reversa de bancada com amina (60 e $100 \mathrm{~g} / \mathrm{t}$ ) e amido (500 e $1000 \mathrm{~g} / \mathrm{t}$ ) em pH 9,5 e 10,7 e três diferentes faixas granulométricas de minério de ferro. Concluíram que, para a faixa grosseira $(150 \mu \mathrm{m}+45 \mu \mathrm{m})$, o aumento na dosagem de amina, mantendo-se constante a dosagem de amido, promoveu aumento do teor de $\mathrm{SiO}_{2}$ no concentrado e diminuição do teor de Fe no rejeito.

Apesar dos inúmeros estudos visando elucidar os mecanismos de adsorção do amido, bem como as possíveis interações entre amido e coletor, poucos trabalhos abordaram as possíveis interações entre esses reagentes na flotação catiônica reversa de minérios de ferro. 0 propósito do presente trabalho é contribuir para o conhecimento das interações entre o amido e o surfatante. Serão analisadas a influência das variáveis de processo pH e relações amido/amina na formação de tais complexos através de ensaios de microflotação e potencial zeta.

\section{REVISÃO BIBLIOGRÁFICA}

Tensoativos, a depender de seu caráter iônico, podem causar formação de complexos de inclusão insolúvel entre suas cadeias hidrocarbônicas e as cadeias glicosídicas da fração amilose, alterando assim as propriedades do amido (Svensson, Gundmundsson \& Eliasson, 1998; Mikus, Hixon, \& Rundle, 1946; Zobel, French, \& Rinkle., 1967). Em geral, tensoativos aniônicos têm maior tendência à formação de complexos que tensoativos catiônicos (Goddard, 1986).

Takagi \& Isemura (1960) também mostraram evidências dessa interação analisando os efeitos do dodecilsulfonato de sódio em soluções de amilose e amilopectina, através da difração de raios-X e medidas de viscosidade. Esta interação foi relacionada à formação de um complexo entre o agente ativo de superfície e a amilose através de sua estrutura helicoidal. Somasundaran (1969) considerou que o amido age na flotação diferentemente de outros depressores, por não prevenir a adsorção de surfatantes na superfície do mineral. Mostrou que o amido diminui o caráter hidrofóbico da calcita através de interações com o oleato, as quais denominou de clatrato. Segundo o autor, essas interações ocorrem através do alojamento do oleato no interior das hélices do amido.

Partridge \& Smith (1972) e Hendrix \& Smith (1972) discutiram a formação de complexos entre a amina e o amido, durante a flotação e a adsorção na superfície mineral. A formação do complexo foi atribuída aos seguintes mecanismos:

i. Atração eletrostática entre $\mathrm{RNH}_{3}$ e a molécula de amido carregada negativamente;

ii. Forças de van der Waals;

iii. Ligações de hidrogênio;

iv. Combinação dos três mecanismos acima.

Segundo Khosla, Bhaoat, Gandhi, \& Biswas (1985), os mecanismos de depressão do amido são:

i. Competição clássica com coletor;

ii. Coadsorção através de combinações binárias depressor/coletor; 
iii. Acomodação do coletor na estrutura helicoidal da amilose.

Somasundaran \& Cleverdon (1985) estudaram interações entre polímero e surfatante catiônico e aniônico em quartzo através de técnicas de adsorção, potencial zeta e flotação. Em pH 6,5 o polímero deprimiu o quartzo, sem diminuir adsorção da amina. O potencial zeta apresentou valores característicos do amido. Por volta do $\mathrm{pH} 10$, em que a amina tem maior atividade superficial, a adsorção do polímero foi negligenciável. A Figura 1 representa a coadsorção do polímero e da dodecilamina sobre a superfície do quartzo, resultando na depressão da flotação do mineral.

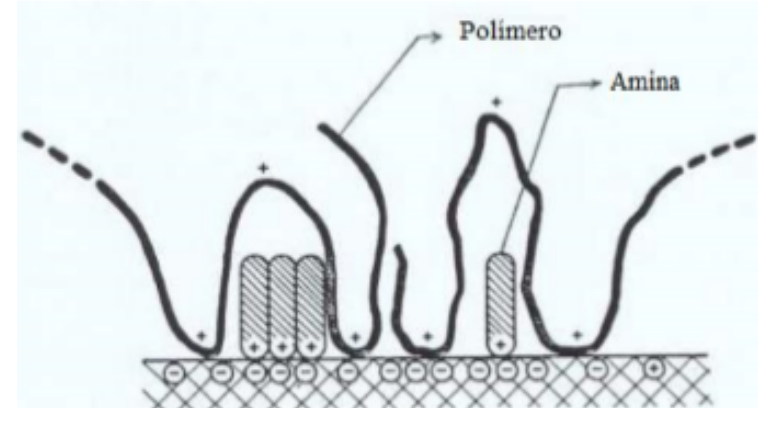

Figura 1: Coadsorção de polímero catiônico de alto peso molecular PAMA e dodecilamina sobre a superfície do quartzo, resultando em depressão da flotação (Somasundaran \& Ramachandran, 1988).

Svensson et al. (1996) estudaram a ligação do dodecilsulfato (SDS) com amilose e amilopectina extraídas do amido de batata. Estimaram que a quantidade de ligações SDS/amilose é muito maior que a quantidade SDS/amilopectina e, no caso da amilose, a atividade superficial do complexo formado aumentou com altas concentrações de surfatante. O complexo SDS/amilose ocorreu através do alojamento das moléculas de SDS no interior das hélices da amilose, conforme mostra a Figura 2. No caso da amilopectina, pode ter havido a ocorrência de pequena contaminação com amilose, mas a hipótese mais aceitável é que as ramificações externas da amilopectina são capazes de formar estes complexos com surfatantes, conforme esquematizado na Figura 3.

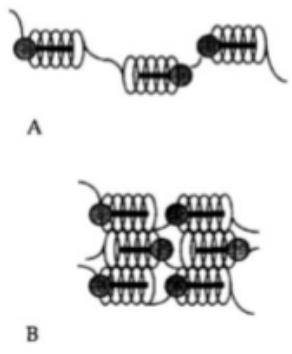

Figura 2: Modelo esquemático para o complexo amilose/SDS (A) com propriedades não cristalinas; (B) com propriedades cristalinas (Svensson et al., 1996).

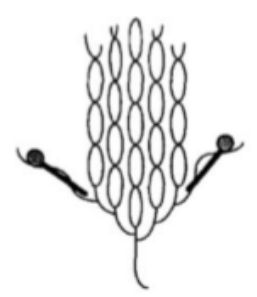

Figura 3: Modelo esquemático para o complexo amilopectina/SDS (Svensson et al., 1996). 
Svensson et al. (1996), nos seus estudos das ligações entre o dodecilsulfato - SDS (surfatante aniônico) e os polissacarídeos amilose e amilopectina, realizaram medidas de tensão superficial utilizando-se a técnica do volume da gota. Quando a tensão superficial de uma solução surfatante é alterada na presença de um polímero, especialmente quando este polímero não é agente tensoativo, pode-se concluir que ocorrem interações ou formação de complexos entre essas substâncias. Através da comparação entre o comportamento da tensão superficial com e sem polímero é possível determinar a capacidade de ligação do surfatante com o polímero. A Figura 4 mostra um diagrama esquemático comparativo da tensão superficial de uma solução de surfatante puro e de uma solução de surfatante associado com polímero.

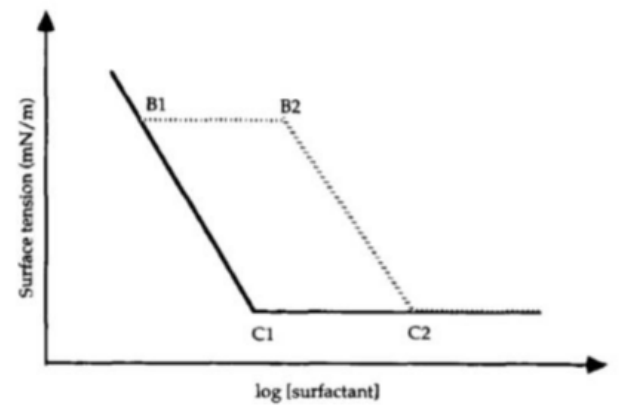

Figura 4: Diagrama esquemático de curvas de pontos de inflexão para surfatantes puros em solução (linha sólida) e solução de surfatante + polímero (linha tracejada). B1 representa o ponto inicial da interação entre surfatante e polímero, e B2 o ponto final, quando o polímero está saturado com surfatante. C1 representa a concentração micelar crítica de surfatante em solução e C2 a CMC da solução de surfatante + polímero (Svensson et al., 1996).

No primeiro estágio, representado pela reta B1-B2 (Figura 4), o surfatante se liga ao polímero, formando complexos de inclusão, que não possuem atividade superficial. Dessa forma, a tensão superficial permaneceu constante nesta região. Após saturação do polímero com surfatante, percebe-se diminuição da tensão superficial, que caracteriza aumento na atividade superficial (Figura 4 B2/C2). Por fim, a partir da concentração C2, um maior empacotamento dos complexos leva à formação de micelas, que faz com que novamente a atividade superficial deixe de ocorrer.

\section{METODOLOGIA}

Foram utilizadas amostras de quartzo e hematita provenientes, respectivamente, de Turmalina-MG e da Mina de Casa de Pedra, localizada em Congonhas (MG). Ambos minerais foram cominuídos em moinho de porcelana e peneirados a úmido, visando obtenção de alíquotas em duas diferentes faixas granulométricas: $-150 \mu \mathrm{m}+75 \mu \mathrm{m}$ para realização de ensaios de microflotação, e $-38 \mu \mathrm{m}$ para caracterização por DRX análise de Potencial Zeta. Os reagentes utilizados no presente trabalho foram $\mathrm{HCl}$ e $\mathrm{NaOH}$ (reguladores de $\mathrm{pH}$ ), eteramina Flotigam EDA como coletor, amido de milho e amidex (amido de milho com elevado teor de amilopectina) como depressores.

Os ensaios de microflotação foram conduzidos seguindo as seguintes etapas de testes:

i. Levantamento de curva de flotabilidade de quartzo em função da dosagem de amina $(\mathrm{pH}=10,5)$, a fim de se obter a dosagem mínima de amina com a qual se obtenha satisfatória flotabilidade do quartzo; ii. Levantamento de curva de flotabilidade de hematita em função da dosagem de amido $(\mathrm{pH}=10,5)$, a fim de se obter a dosagem mínima de amido com a qual se 
obtenha satisfatória depressão da hematita; iii. Levantamento de curvas de flotabilidade de quartzo em função do $\mathrm{pH}$ para diferentes dosagens de amina e amido de milho, a fim de verificar as dosagens e valores de $\mathrm{pH}$ que favorecem a formação dos complexos amina/amido. iv. Levantamento de curvas de flotabilidade de quartzo em função do $\mathrm{pH}$ para diferentes dosagens de amina e amidex, a fim de verificar as dosagens e valores de $\mathrm{pH}$ que favorecem a formação dos complexos amina/amido.

As medidas de Potencial Zeta restringiram-se à amostra de quartzo, e ocorreram nas seguintes etapas:

i. Quartzo condicionado com $80 \mathrm{mg} / \mathrm{L}$ de amina e $80 \mathrm{mg} / \mathrm{L}$ de amina e amido (condição com evidência de formação de clatratos).

\section{RESULTADOS E DISCUSSÃO}

Após a realização das análises de difratometria de raios- $X$, comprovou-se que as amostras de quartzo e hematita possuíam elevado grau de pureza.

\subsection{MICROFLOTAÇÃO}

Os resultados dos ensaios de microflotação visando levantar a dosagem mínima de amina necessária para obtenção de máxima flotabilidade dos minerais hematita e quartzo sem a presença de depressor estão apresentadas na Figura 1. Observa-se que, na ausência de amido, a amina adsorve-se tanto na superfície do quartzo quanto na superfície da hematita. Entretanto, no caso do quartzo, o máximo de flotabilidade foi atingido em concentração de $4 \mathrm{mg} / \mathrm{L}$ de amina, enquanto que para a hematita apenas em concentrações superiores a $10 \mathrm{mg} / \mathrm{L}$. O patamar de máxima flotabilidade de ambos os minerais foi de aproximadamente $95 \%$. A menor concentração de amina necessária à obtenção de máxima flotabilidade do quartzo deve-se à maior afinidade do íon coletor com o quartzo que, em toda faixa de $\mathrm{pH}$, apresenta carga superficial mais negativa que a carga superficial da hematita. Vale ressaltar que, assim sendo, na ausência de depressor, torna-se impossível alcançar adequada seletividade na flotação catiônica reversa de minérios de ferro.

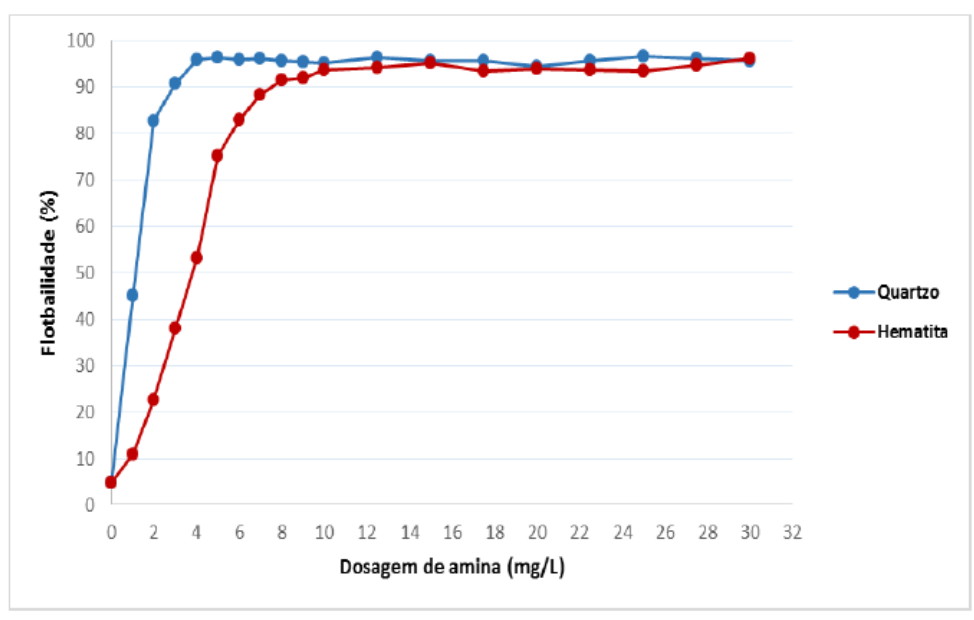

Figura 1: Flotabilidade de hematita e quartzo em função da concentração de amina, $\mathrm{pH}=10,0$. 
Posteriormente foi investigada a dosagem mínima de amido necessária para promover a máxima depressão da hematita. Foram comparados os efeitos do amido de milho do tipo maisena (75\% de amilopectina) e amidex (98\% de amilopectina). Os resultados encontram-se na Figura 2. Os resultados apresentados mostram que o amidex apresenta melhor ação depressora sobre a hematita, quando comparado com a maisena. Este resultado pode ser explicado pelo fato da amilopectina apresentar maior peso molecular em relação à amilose, sendo dessa forma um floculante mais poderoso.

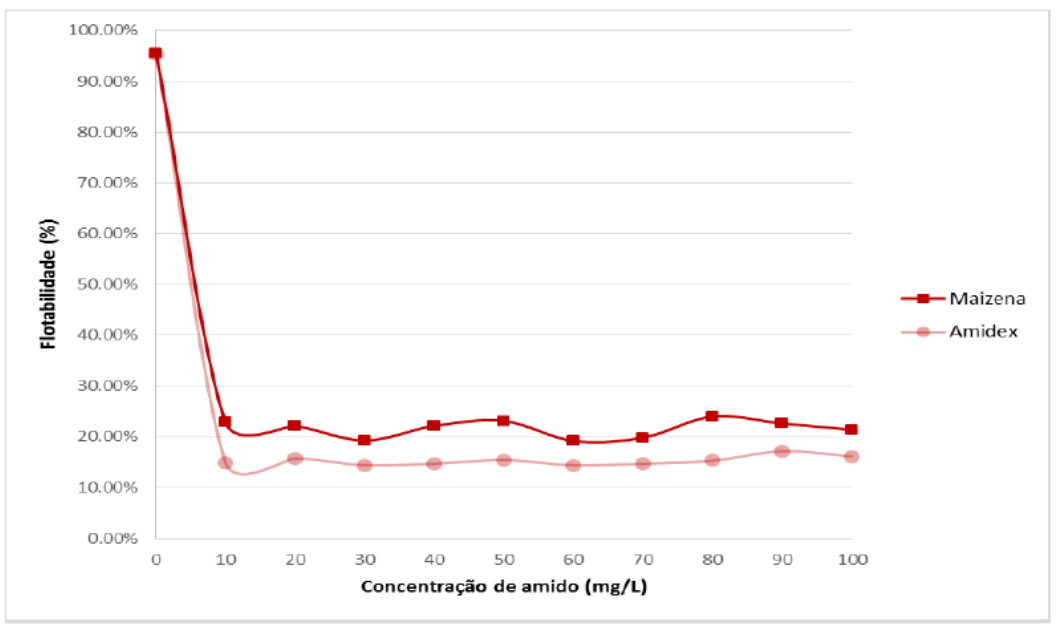

Figura 2: Flotabilidade da hematita em relação à concentração dos depressores, com amina $(10 \mathrm{mg} / \mathrm{L}) \mathrm{em}$ pH=10,0.

Para verificar a influência das concentrações de amina e amido do tipo maisena sobre a depressão do quartzo, foram realizados diversos ensaios, variando-se as dosagens de ambos os reagentes, cujos resultados encontram-se na Figura 3. Observa-se claramente que, quanto maiores as dosagens de maisena e amina, maior a tendência de depressão do quartzo.

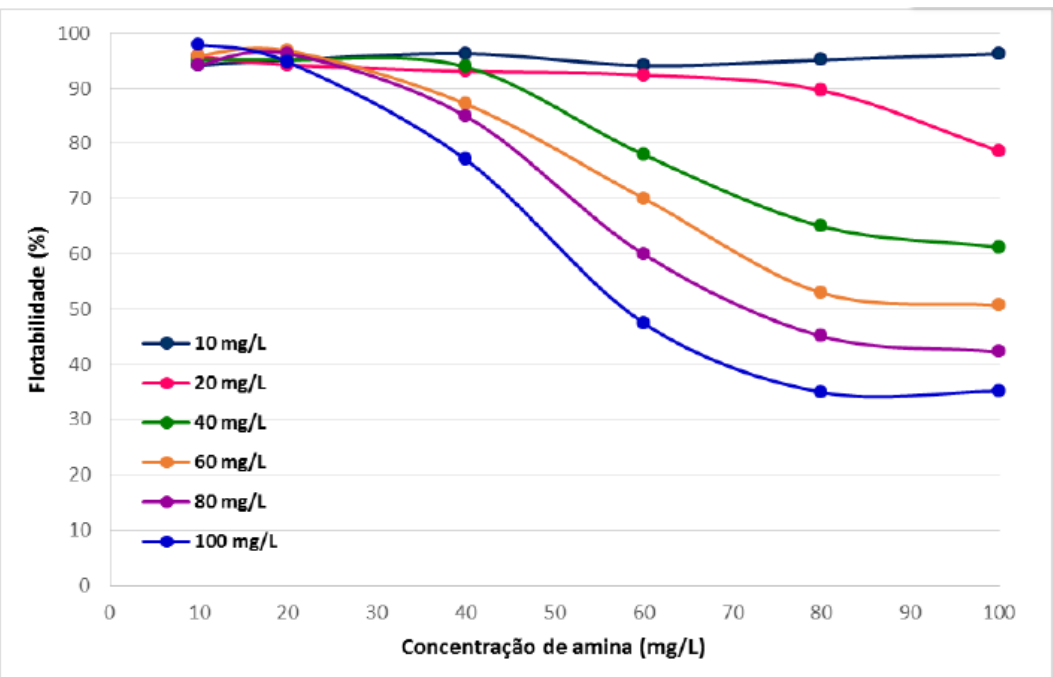

Figura 3: Flotabilidade de quartzo em função de diferentes concentrações de amina e maisena, pH=10.

Quando se realizou ensaios de microflotação utilizando amina e amidex nas mesmas condições que os ensaios com Amido, não se observou em nenhuma condição depressão do quartzo. Tal resultado sugere que, assim como afirmado por diversos autores (Somasundaran, 1969; Takagi \& Isemura, 1960), a formação de clatratos ocorre principalmente devido ao alojamento da molécula de surfatante no interior das hélices da amilose. 
Por fim, com a finalidade de se verificar como o $\mathrm{pH}$ pode influenciar a formação dos clatratos, foram realizadas microflotações, mantendo-se constantes as concentrações de amina e amido ( $80 \mathrm{mg} / \mathrm{L}$ ), e variando-se apenas o tipo de amido, uma vez que, conforme mostrado na etapa anterior da microflotação, apenas a maisena foi capaz de diminuir a flotabilidade do quartzo. A Figura 4 mostra os resultados obtidos. A forte dependência da queda na flotabilidade do quartzo com o pH sugere que ao menos uma variável dependente do $\mathrm{pH}$ desempenha papel importante no mecanismo de formação dos clatratos. Na presente investigação pode haver contribuição das seguintes variáveis: concentração de amina ionizada ou molecular, ionização dos constituintes do amido, conformação da amilose e carga superficial do quartzo. De acordo com Somasundaran (1975) e Banks \& Greenwood (1972), a amilose apresenta estrutura helicoidal principalmente em condições alcalinas e na presença de um agente complexante, que constituem exatamente as condições utilizadas no presente trabalho.

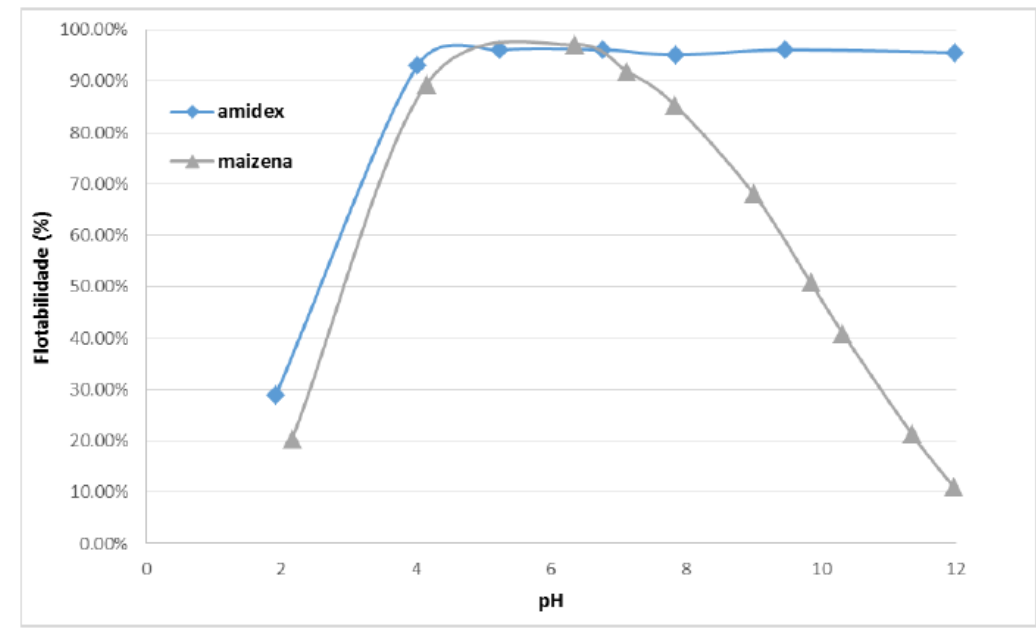

Figura 4: Flotabilidade do quartzo em função do pH e do tipo de amido ( $80 \mathrm{mg} / \mathrm{L}$ de amina e $80 \mathrm{mg} / \mathrm{L} \mathrm{de} \mathrm{amido).}$

\subsection{POTENCIAL ZETA}

A presente etapa de ensaios foi realizada com o intuito de se determinar as possíveis influências da formação de clatratos no potencial zeta do quartzo, a fim de se elucidar os mecanismos de adsorção do amido e amina na interface quartzo-solução. Observa-se, com base na Figura 6, que na condição que favoreceu a formação de clatratos o potencial zeta do quartzo tornou-se menos negativo para $\mathrm{pH}$ superior a 5 , que coincidiu com o pH em que observou-se diminuição da flotabilidade do quartzo, conforme mostram os resultados das microflotações. Uma hipótese que pode explicar o resultado observado na Figura 6 é que a formação de complexos entre amido e amina pode levar a um maior grau de adsorção da amina sobre a superfície do quartzo, contribuindo assim com diminuição do módulo do potencial zeta observada. Uma outra observação que se pode fazer com relação à figura é que, como não houve diminuição do potencial zeta, a amina não deixou de se adsorver no quartzo para formar os complexos em solução. Dessa forma, o amido deve de fato coadsorver-se na interface quartzosolução, em associação com a amina. 


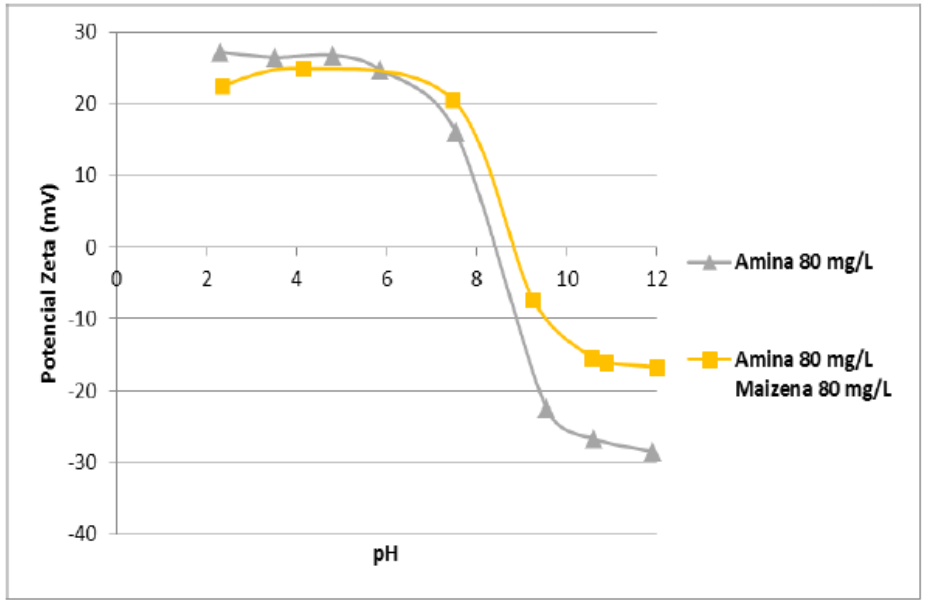

Figura 5: Potencial zeta do quartzo em função do pH e da concentração de reagentes $(0,001 \mathrm{M}$ de $\mathrm{NaCl})$.

\section{CONCLUSÃO}

A coadsorção de amido e amina na interface quartzo-solução pode ser explicada em termos do clatrato formado entre o amido em sua forma helicoidal e a amina adsorvida no interior das hélices. Em escala de microflotação, observou-se que o aumento das dosagens de amina e amido favoreceu a formação dos clatratos, já que a flotabilidade do quartzo chegou a cair de $95 \%$ a 35\% como consequência do aumento da dosagem de amina. A diminuição da flotabilidade do quartzo se pronunciou mais em níveis mais altos de $\mathrm{pH}$. Não houve queda na flotabilidade quando se utilizou amidex como depressor de hematita. Verificou-se aumento do potencial zeta do quartzo com a adsorção de maisena e amina em condições que favoreceram a formação de clatratos, se comparado com as mesmas condições, sem adsorção de maisena. Assim, acredita-se que a queda na flotabilidade do quartzo ocorra devido à coadsorção de amido e amina na interface quartzo-solução.

\section{REFERÊNCIAS}

Banks, W., \& Greenwood, C. T. (1972) The conformation of amylose in alkaline salt solution. In: Carbohydrate Research; 229-234;

Goddard, E. D. (1986) Polymer-surfactant interaction. Part I. Uncharged water soluble polymers and charged surfactants. In: Colloids Surfaces, v. 19, 255-300;

Hendrix, D. W., \& Smith, G. W. (1972) Interaction of amine and starch in quartz flotation. In: Canadian Metallurgical Quarterly, v. 11, 303-308;

Khosla, N. K., Bhaoat R. P., Gandhi, K. S., \& Biswas A. K. (1985) Calorimetric and other interaction studies on mineral-starch adsorption systems. In: Colloids and Surfaces, v. 8, 321-336;

Lima, N. P., Valadão, G. E. S., \& Peres, A.E.C. (2013) Effect of amine and starch dosages on the reverse cationic flotation of iron ore. In: Minerals Engineering, Elsevier Science Publishers n45., 180-184;

Mikus, F. F., Hixon, R. M., Rundle, R. E. (1946) The complexes of fatty acids with amylose. Journal of the American Chemical Society, v.68, 1115-1123;

Partridge, A. C., \& Smith, G. W. (1971) Flotation and adsorption characteristics of hematite- 
dodecylamine-starch system. In: Canadian Metallurgical Quarterly, v. 10, 229-235;

Somasundaran, P. (1969) Adsorption of starch and oleate and interaction between them on calcite in aqueous solutions. In: Journal of Colloid and Interface Science, v. 31, 557-565;

Somasundaran, P. (1975) Interfacial chemistry of particulate flotation. Henry Krumb School of Mines, Columbia University, New York;

Somasundaran, P., Cleverdon, J. (1985) A study of polymer/surfactant interaction at the mineral solution interface. In: Colloids and surfaces, v.13, 73-85;

Svensson, E., Gundmundsson, M., Eliasson, A. C. (1996) Binding of sodium dodecylsulphate to starch polyssacharides quantified by surface tension measurements. In: Colloids and Surfaces B, v.6, 227-233;

Svensson, E., Autio, K. M., \& Eliasson, A.C. (1998) The effect of sodium dodecylsulphate on gelatinization and gelation properties of wheat and potato starches. In: Food hydrocolloids, v.12, 151-158;

Takagi, T., \& Isemura, T. (1960) Interaction of surface active agents with amilose. Chemical Society of Japan, v. 33, 437-441;

Zobel, H. F., French, A. D., \& Hinkle, M. E. (1967) X-ray diffraction of oriented amylose fibers. II Structure of amylose. In: Biopolymers, v. 5, 837-845. 